\title{
Radiation Therapy During the COVID-19 Pandemic: Experience from Beijing, China
}

\author{
WEIPING WANG*, XIAOLIANG LIU*, FUQUAN ZHANG, JIE QIU and KE HU \\ Department of Radiation Oncology, Peking Union Medical College Hospital, \\ Chinese Academy of Medical Sciences \& Peking Union Medical College, Beijing, P.R. China
}

\begin{abstract}
Background: Coronavirus disease 2019 (COVID19) is now a global pandemic. It is unclear to radiotherapy practitioners how to carry out radiotherapy during the epidemic. Patients and Methods: After the outbreak of COVID-19, our Institute established measures for the prevention and control of COVID-19, and continues to treat patients according to these measures. The Radiotherapy Department has been divided into a clean zone and a semicontaminated zone, and corresponding personal protective equipment is used in these zones. The temperature of patients and their escorts, and history of fever are assessed daily. Special procedures are performed during radiotherapy setup and intracavitary brachytherapy. Results: Over a period of 2 months, 655 patients were treated in the Department. Sixteen patients with fever were identified and no patient undergoing radiotherapy or medical staff have been infected with COVID-19. Conclusion: Our protective measures were found to be effective and can be used as a reference in places where COVID-19 situations are not markedly serious.

Coronavirus disease 2019 (COVID-19) is now a global
\end{abstract}

This article is freely accessible online.

*These Authors contributed equally to this study.

Correspondence to: Fuquan Zhang, Department of Radiation Oncology, Peking Union Medical College Hospital, Chinese Academy of Medical Sciences \& Peking Union Medical College, No. 1 Shuaifuyuan Wangfujing Dongcheng District, Beijing 100730, PR China. Tel: +86 01069155485, e-mail: zhangfuquan3@sina.com; Jie Qiu, Department of Radiation Oncology, Peking Union Medical College Hospital, Chinese Academy of Medical Sciences \& Peking Union Medical College, No. 1 Shuaifuyuan Wangfujing Dongcheng District, Beijing 100730, PR China. Tel: +86 01069155481, email: qj_ww@139.com; Ke Hu, Department of Radiation Oncology, Peking Union Medical College Hospital, Chinese Academy of Medical Sciences \& Peking Union Medical College, Shuaifuyuan Wangfujing Dongcheng District, Beijing 100730, PR China. Tel.: +86 01069155487, e-mail: huk@pumch.cn

Key Words: COVID-19, radiotherapy, personal protective equipment. pandemic. According to World Health Organization statistics, there have been 3.6 million cases and nearly 25,000 deaths around the world as of May 7, 2020 (1). The disease not only poses a huge challenge to medical health systems in various countries but also creates enormous obstacles in the diagnosis and treatment of patients with other diseases, such as tumors, during the COVID-19 outbreak. As an important oncotherapy approach, radiotherapy has certain particularities. Some patients with tumors can be cured by radical radiotherapy or chemoradiotherapy, which are life-saving approaches. Any delay or interruption of this therapy can negatively affect its efficacy. Currently, how to carry out radiotherapy during the epidemic is unclear to radiotherapy practitioners in many countries, especially in those where COVID-19 is prevalent. Recently, a cluster of infections with severe acute respiratory syndrome coronavirus 2 , the virus responsible for COVID19, occurred in the Radiation Oncology Department of a hospital in Turkey. Thirteen out of 18 radiation oncologists, one physicist, one technician and one nurse were infected with COVID-19. Under such circumstances, radiotherapy services in this hospital were nearly shut down (2).

In China, Wuhan was the center of the COVID-19 outbreak. At the time of writing, there were over 50,000 confirmed cases in this city, and more than 2,500 patients had died. Radiotherapy experience during the COVID-19 outbreak was previously reported by Hubei Cancer Hospital in Wuhan $(3,4)$. Their procedure can be used in most affected areas, such as Wuhan. However, the outbreak in other regions of China and the vast majority of places around the world is not as serious as that in Wuhan. In less affected areas, an emphasis should be placed on, for example, investigating how to scientifically prevent, control and treat patients with existing conditions, avoid wasting medical supplies and avoid causing panic among patients and medical staff at the same time.

It has been reported to date that the cumulative number of confirmed cases has reached 593 in Beijing, China, and nine patients with COVID-19 have died. Peking Union Medical College is a comprehensive hospital in Beijing. The number of patients treated with radiotherapy at our hospital had reached 
approximately 350-450 daily before the COVID-19 outbreak. After the disease outbreak, the hospital has continued to conduct radiotherapy. The number of patients remained large, and most patients came from various places in China and were treated as outpatients at our Institute. As a result, patients and medical staff have been subjected to a dramatic risk of being exposed due to high patient mobility since the outbreak of COVID-19. The present study was carried out with a focus on the measures preventing and controlling COVID-19 taken at our hospital over the past 2 months, as well as their corresponding effects. This article is expected to provide a reference for radiotherapy centers in other areas, especially areas with similar COVID-19 situations.

\section{Patients and Methods}

Formulation of COVID-19 prevention and control scheme. If radiotherapy services are to be provided during an outbreak of COVID-19, it is suggested that a COVID-19 prevention and control scheme should be formulated in advance. The purpose of such a scheme is to guarantee a normal radiotherapy process for patients with cancer on the premise of ensuring scientific prevention and control. After the COVID-19 outbreak, our hospital released the "COVID-19 Prevention and Control System \& Standard Operation Procedures of Peking Union Medical College Hospital". On this basis, a COVID-19 prevention and control scheme for the Radiotherapy Department was also prepared. The details are described as follows.

Training and protective measures for working staff. Staff training: As required, all doctors, nurses, medical physicists and therapists learned the latest COVID-19 diagnosis and treatment schemes, as well as the corresponding prevention and control measures. Only those who were proven to be qualified by relevant tests were permitted to work in the Radiotherapy Department. In addition, none of the working staff were allowed to participate in any public events, such as gatherings, except those required by their job. Staff members with nonmedical backgrounds (e.g. cleaners, and security guards) were provided with additional training on nosocomial infection prevention and control about COVID-19 provided by the Radiotherapy Department.

Zone division and corresponding personal protective equipment (PPE): The Radiotherapy Department was partitioned into a clean area and a semi-contaminated area. Both were disinfected according to the guidelines described below.

(i) Clean area: Staff lounge; locker room; rest room; and other areas where the staff can eat. No PPE that has been used should be present. With the goal of preventing cross-infection, staff in a clean area must correctly wear masks when there are two or more members present.

(ii) Semicontaminated area: Consulting room; treatment room; mold room; medical physics and dosimetry office; physician's office; waiting area; and, other areas where items, such as instruments, devices and linen, that have been used by patients can be handled. Please note that these areas may be contaminated by blood, secreta and pathogenic microorganisms. A Level 1 protection standard should be applied here. Uniforms, disposable caps and disposable medical surgical masks, latex gloves and disposable oversleeves should be used in this area. Moreover, protective goggles or face shields may be worn during intracavitary brachytherapy (ICBT), oral/nasal examinations, or other operations that may lead to an exposure risk to blood, respiratory droplets and body fluids. In the early stage of the COVID-19 outbreak, disposable ordinary masks are recommended to medical physicists who are barely exposed to patients with COVID-19 for the purpose of sparing medical supplies.

Scheduling requirements: Radiation therapists are divided into three fixed shifts on a daily basis; each shift is on duty for 5-6 hours every day. In this way, the range of infections that may be caused by potentially infected persons can be reduced to the greatest extent.

Quarantine requirements: Before entering their respective Departments, the working staff must take their body temperature. If symptoms such as fever, dry cough and pharyngalgia are found, they must undergo quarantine examinations according to the screening requirements of the hospital. In addition, symptomatic staff are prohibited from working.

Disinfection of the environment and equipment. Floor disinfection: Floor disinfection is performed by spraying $500 \mathrm{mg} / \mathrm{l}$ chlorinecontaining disinfectant, and the required duration of action should be greater than 30 minutes; after that, the floor surface should be scrubbed with clean water. For semi-contaminated zones, at least one additional disinfection is required per day, and the disinfection frequency may be proportionally increased to conform with patient flow. Regarding the clean area, it is disinfected at a minimum of once a day.

Air disinfection: Primarily, air disinfection is conducted by means of ventilation at least two or three times a day. In addition, the duration of ventilation should be no less than 30 minutes each time. Based on the density of patients awaiting diagnosis, the number of times ventilation is allowed should be increased proportionally. If permitting, doors and windows can also normally be left open for ventilation. Rooms without proper ventilation are forbidden from being used for treatment or diagnosis. After treatment, the corresponding room may be exposed to light from ultraviolet irradiators for 1 hour to disinfect the indoor air and object surfaces. The ventilation system should be cleaned and disinfected once a month. When necessary, air disinfection may be carried out. When there are no people in the room, disinfection can be performed by spraying $500 \mathrm{mg} / \mathrm{l}$ chlorine-containing disinfectant, or an air disinfecting machine should be selected when someone is in the room.

Body surface disinfection: The semi-contaminated area should be principally wiped with disinfectant wipes containing alcohols, with $75 \%$ ethyl alcohol or $500 \mathrm{mg} / \mathrm{l}$ chlorine-containing disinfectant after cleaning. Object surfaces that are frequently exposed, such as seats for patients awaiting diagnosis, wall surfaces, guard rails, handrails, doorknobs and nurse's stations should be disinfected at least twice a day. In terms of walls and ceilings that are seldomly touched, disinfection should be performed once a month at minimum. Clean areas should be wiped with clean water, $75 \%$ ethyl alcohol or 500 $\mathrm{mg} / \mathrm{l}$ chlorine-containing disinfectant if necessary.

Equipment disinfection: Large medical equipment, including accelerators, computed tomography (CT) simulators, conventional simulators and ICBT machines, their enclosures and treatment couches should be disinfected by being wiped with $75 \%$ ethyl alcohol or wipes that contain $75 \%$ ethyl alcohol. No atomization devices are permitted to be sprayed with flammable disinfectants such as ethyl alcohol for disinfection. During wiping, it should be 
ensured that no disinfectant flows into the interior of equipment, as this may lead to equipment issues or electric shock accidents. Regarding peripheral equipment (e.g. computer monitors, keyboards, printers and code scanning facilities), they should be wiped and disinfected with $75 \%$ ethyl alcohol or wipes containing $75 \%$ ethyl alcohol.

Therapy management for patients with cancer. Clinics: In addition to regular consultations in clinics, an online consultation service is also provided in the hospital during the COVID-19 pandemic. Patients are able to seek physicians' advice through an application from Peking Union Medical College Hospital. Those who need to visit the physician's office based on the physician's opinion can make an appointment for a corresponding regular consultation in the clinic.

Indications for radiotherapy: (i) The indications for radiotherapy should be rigorously defined. In the event where a patient has a definite indication, the corresponding radiotherapy is still recommended. Moreover, the conditions and previous treatment of the patient are analyzed. If radiotherapy can be conducted at a later date, it should be postponed before simulation and treatment occur.

(ii) The temperature of the patients and their escorts and any history of fever, exposure, and travel within the previous 2 weeks are assessed. Patients who have a fever or relevant medical history are required to undergo COVID-19 screening at the fever clinic. Patients with COVID-19 or suspected COVID-19 cannot receive radiotherapy. The diagnostic criteria for confirmed and suspected COVID-19 cases have been elaborated in China's COVID-19 Diagnosis and Treatment Scheme (5).

Patients in the radiotherapy area: (i) The temperature of patients is assessed at the door of the radiotherapy area. Only patients without fever are allowed to enter the Radiotherapy Department. The patients and their escorts must wear masks and shoe covers and disinfect their hands throughout the process. A distance of no less than $1 \mathrm{~m}$ is kept between people, and conversations should be avoided.

(ii) The number of patients and escorts in the radiotherapy area is restricted. An appointment system is implemented, and the time of therapy is relatively fixed on a daily basis. For patients requiring company, the number of escorts for a patient should be restricted. For the purpose of alleviating infection risk inside the Department, patients are allowed to enter the waiting area half an hour before their appointment time.

(iii) Certain measures should be taken to ensure that there is only one patient in the consultation room at a time.

Radiotherapy setup: (i) Patients are required to fill out a form about their history of fever, exposure, living and traveling details each day.

(ii) A cloth or disposable sheet should be provided to each patient. Before and after exposure to any patient, the medical staff must wash their hands or disinfect their hands with a quick disinfecting solution. When necessary, disposal latex gloves may be worn. Moreover, disinfection of the hands must be conducted before wearing or removing gloves.

(iii) When positioning patients with a risk for blood, body fluids and secreta splatter, medical staff may wear a pair of protective goggles or a face shield

ICBT: (i) Equipment and environmental disinfection, associated device preparation, disposable article replacement, and hand disinfection must be carried out in strict accordance with relevant standard procedures. (ii) The physician or nurse performing applicator placement may wear a general isolation gown, medical surgical mask, nonwoven cap and protective goggles.

(iii) A transfer route should be planned in advance. Transferred patients should wear masks and avoid contact with populations awaiting diagnosis to the greatest extent. In addition, stopover should be avoided during transfer.

(iv) For operators responsible for taking out the applicator, the corresponding protective measures are the same as those for applicator placement.

Patient management and follow-up: During treatment, patients should undergo blood tests on a weekly basis, and the corresponding physician may inform them about their illness status through SMS messages, WeChat, telephone or an application from the Peking Union Medical Hospital. Patient follow-up after treatment is carried out online. If necessary, the patient can make an appointment through the outpatient service.

Emergency plan for fever. Under the circumstance that the body temperature of a patient exceeds $37.3^{\circ} \mathrm{C}$, they need to seek a physician's advice at the fever clinic and undergo both a routine blood test and a chest CT. In conjunction with the basic conditions of the patient and relevant examination results, the reason for the fever can be determined. When necessary, a COVID-19 nucleic acid assay of the respiratory tract can be conducted by the fever clinic. Once a nucleic acid assay is required, the working staff exposed to the patient on that day are not allowed to leave the hospital before the nucleic acid assay result is reported as being negative. If confirmed to be infected with COVID-19, patient will be transferred to a designated hospital and the working staff and other patients who were in close contact with them during the previous 14 days must undergo a 14-day quarantine. In this scenario, the Department of Radiotherapy should be thoroughly disinfected. Medical waste exposed to the confirmed patient should be packed in two packaging bags specifically used for medical waste according to requirements of the hospital and then sealed after disinfection with a disinfectant. In addition, the bags should be placed and sealed in a disposable compression-resistant carton. After sealing, the carton should not be opened again.

Patients. We retrospectively analyzed patients treated at our Institute during the Wuhan lockdown from January 23, 2020 to March 31, 2020. Information on basic characteristics of patients, daily treatment conditions, and the management of patients with brain metastasis, cervical cancer, pediatric tumors and fever were collected.

\section{Results}

General situation of patients during the COVID-19 pandemic. A total of 655 patients were treated with radiotherapy at our Institute. Among them, 297 were admitted during the COVID-19 pandemic, while the treatment for others was initiated before the pandemic started. A total of 655 patients were treated with radiotherapy at our Institute. Among them, 297 were admitted during the COVID-19 pandemic, while the treatment for others was initiated before the pandemic started. Of these 297 patients, 141 were patients local to Beijing (47.5\%), over 50\% came from other places than Beijing, 


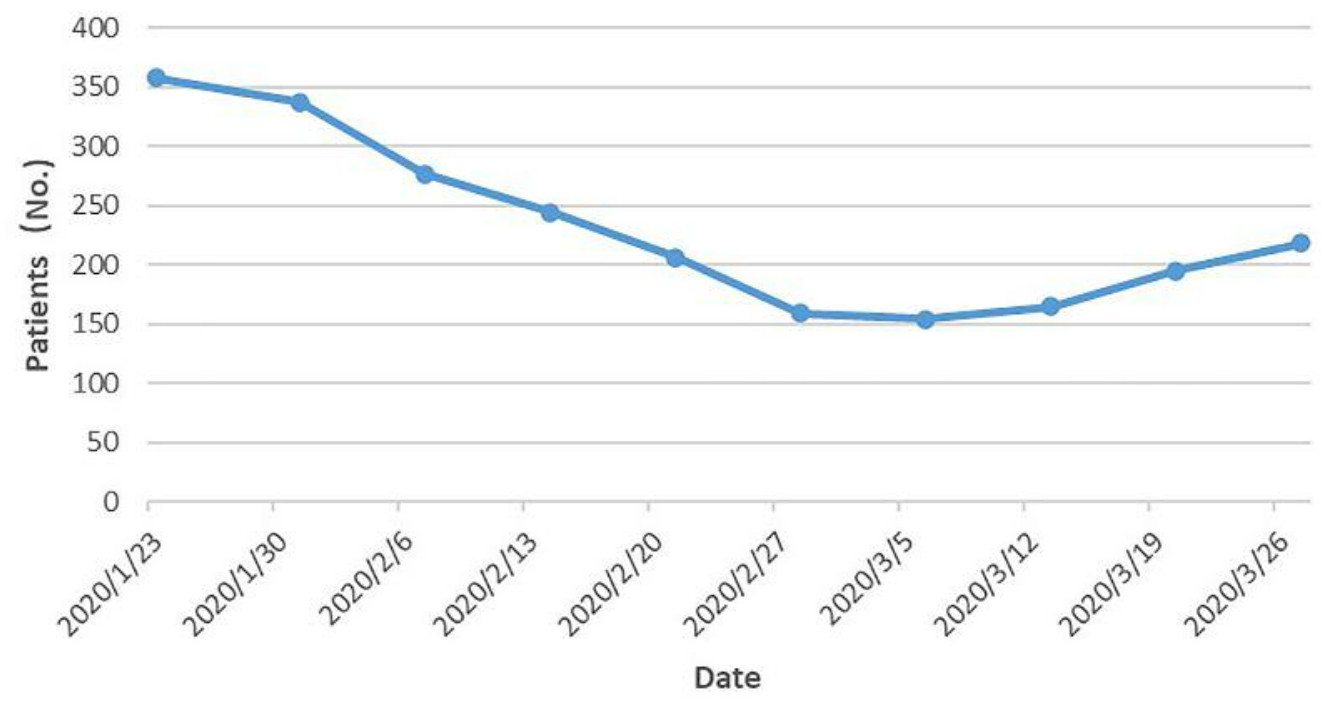

Figure 1. Daily variations in the number of patients treated during the COVID-19 pandemic.

while three patients $(1.0 \%)$ came from Hubei, however, these three patients all arrived at Beijing for medical consultations before the Wuhan lockdown and had not left Beijing since then. Among the 297 newly admitted patients, 88 patients had gynecological tumors, which accounted for the highest percentage $(29.6 \%)$, followed by breast cancer $(74,24.9 \%)$, digestive system neoplasms $(33,11.1 \%)$, pediatric tumors $(31$, $10.4 \%)$, head and neck neoplasms $(25,8.4 \%)$, lung cancer $(22,7.4 \%)$, prostatic cancer $(10,3.4 \%)$ and other cancer types $(14,4.8 \%)$.

Daily treatment conditions of patients during the COVID-19 pandemic. On January 23, 2020, the first day of the Wuhan lockdown, 358 patients were treated at the Department of Radiotherapy of our hospital. As the situation around preventing and controlling COVID-19 changed, the emergency response grade rose to grade 1 in various places of China. Consequently, most patients with cancer postponed their treatment, so that the number of patients treated at the Department began to gradually decline day by day and reached its minimum value (154 cases) on March 6, 2020. Subsequently, this number went up progressively each day. As of March 27, 2020, the number of patients receiving treatment at the Department had increased to 218 per day. The details are illustrated in Figure 1.

At present, there are six linear accelerators in the Department, including two Tomotherapy units, one VARIAN Halcyon, one VARIAN Truebeam, one Siemens Artist and one ELEKTA Versa HD devices. Before the outbreak of COVID-19, a total of five accelerators can be run normally. After the outbreak, only three accelerators were used for treatment due to a gradual decrease in the number of patients. In addition, each patient queuing for treatment was allowed to enter the therapy area and receive treatment only after the former patient had left the area completely after their own treatment had ended. Therefore, it is expected that these patients were protected from gathering and avoided queuing to the greatest extent possible; in addition, their exposure time was minimized. For this reason, the treatment efficiency of the Department slightly dropped. Taking the VARIAN Halcyon accelerator as an example, it was used to treat 7-8 patients in an hour before the COVID-19 pandemic; now, that number has declined to approximately five patients per hour.

Management of patients with brain metastasis, cervical cancer, pediatric tumors and fever. During the outbreak of COVID-19, nine patients with brain metastasis were received and treated at the Department, including three acute and severe cases. On the basis of preventing and controlling COVID-19, the radiotherapy schedule was expedited for these patients. After treatment, their symptoms significantly improved.

Moreover, cervical cancer is our specialty. For patients undergoing radical radiotherapy, ICBT should be conducted 4-6 times during treatment. During this period, 504 cycles of ICBT were provided in total, that is, nine cycles of ICBT each day.

Pediatric tumors are another specialty of the Department. Pediatric patients generally cooperate poorly with physicians, and their parents are anxious in most cases. During the COVID-19 pandemic, this situation has worsened. Thus, 
sufficient psychological counseling was provided for the parents of pediatric patients. In addition, one escort was allowed to accompany each pediatric patient after the COVID-19 outbreak. As a result, radiotherapy for 51 pediatric patients was successfully completed, including 20 who were under treatment and 31 who were newly admitted.

Additionally, the body temperature of each patient was measured each day during the COVID-19 pandemic. Through screening, 16 patients with fever $\left(\geq 37.3^{\circ} \mathrm{C}\right)$ were identified, and all of them were transferred to the fever clinic. Based on preliminary screening in the clinic (including epidemiological, hematological and imaging examinations), 15 patients were believed to be suffering from neoplastic fever not related to COVID-19. After symptomatic treatment, these patients were provided with further radiotherapy. The other patient had cervical cancer with pulmonary metastasis. The imaging examination showed a ground-glass shadow in the left lung, and further nucleic acid assays to screen for COVID-19 confirmed negative results. Radiotherapy was continuously provided to the patient. After the nucleic acid assay was performed, however, radiotherapy was suspended before obtaining the testing result. In addition, all staff and other relevant personnel who were exposed to this patient were subjected to an in-situ quarantine according to the requirements of the hospital.

No patients undergoing radiotherapy or working staff were infected with COVID-19 during this challenging time period.

\section{Discussion}

During the COVID-19 pandemic, it is of equal importance to guarantee other basic medical services in addition to being concerned about COVID-19. As a therapy used to save lives, tumor radiotherapy should be provided and guaranteed. During the COVID-19 outbreak, radiotherapy should be arranged on the premise of following specific plans and taking protective measures. Now, the entire world is running short of protective supplies, such as disposable isolation gowns and N95 masks. Many countries have even failed to ensure PPE for medical staff involved in COVID-19 treatment. Considering this, protection for people engaged in radiotherapy should be limited to a certain level, and overprotection should be avoided, especially in regions where the COVID-19 situation is less serious. It has been reported by the Hubei Cancer Hospital that during patient positioning or treatment, grade 3 protection should be worn by medical staff in contaminated areas (e.g. treatment vaults, LINAC console areas, and CT/conventional simulation room). According to stipulations on grade 3 protection, the relevant protective articles consist of disposable surgical caps, N95 respirators, protection suits, protection goggles, disposable gloves, and disposable shoe covers (3). This is a reasonable measure in Wuhan and other places with severe COVID-19 situations. However, protection at such a level may waste considerable medical supplies in places where the COVID-19 situation is not quite as serious, especially under the circumstance of a medical supply shortage. At the Department of Radiotherapy here, grade 1 protection was selected for the working staff. These staff members were only equipped with protective suits, disposable surgical caps and disposable medical surgical masks. When necessary, they were allowed to put on latex gloves and disposable nonwoven oversleeves, and protective goggles or shields were only recommended for cases where the spattering of blood, respiratory droplets or body fluids might have taken place. Moreover, no disposable isolation gowns or N95 masks were suggested for use.

Although there is only a small population confirmed with COVID-19 in Beijing, we are still confronted with a huge challenge. Firstly, ours is a general hospital with an established fever clinic. At this clinic, there are multiple patients who have been confirmed to have COVID-19. Secondly, most patients of the hospital are from other places outside Beijing, and some even came from Hubei Province, which had the most severe COVID-19 outbreak. Furthermore, these patients sought doctors' advice through outpatient services. Consequently, the patients may stay in contact with the outside world during treatment. Thirdly, each accelerator in the hospital is used to treat a larger number of patients when compared with that in developed countries. Thus, patient clustering is highly likely to occur. Over a 2-month period, 655 patients were treated at the Department. Thanks to our rigorous protective measures, no patients undergoing radiotherapy or medical staff have been infected with COVID-19. This indicates that these protective measures are effective and can be used as a reference in places where COVID-19 situations are not markedly serious.

\section{Conflicts of Interest}

The Authors declare that they have no conflicts of interest in regard to this study.

\section{Authors' Contributions}

Concept and study design: FZ, JQ, KH; Data collection: WW, XL; Writing-original draft preparation: WW; Formal analysis: XL; Writing, review and editing: FZ.

\section{References}

1 World Heath Organization. Coronavirus Disease (COVID-19) Outbreak Situation. Available at: https://www.who.int/ emergencies/diseases/novel-coronavirus-2019 [Last accessed on May 7th 2020] 
2 Gemici C and Yaprak G: COVID-19 outbreak in a major radiation oncology department; Which lessons should be taken? Radiother Oncol, 2020. PMID: 32342865. DOI: 10.1016/ j.radonc.2020.03.044

3 Wei W, Zheng D, Lei Y, Wu S, Verma V, Liu Y, Wei X, Bi J, Hu D and Han G: Radiotherapy workflow and protection procedures during the Coronavirus disease 2019 (COVID-19) outbreak: Experience of the Hubei Cancer Hospital in Wuhan, China Radiother Oncol, 2020. PMID: 32342870. DOI: 10.1016/ j.radonc.2020.03.029

4 Wu S, Zheng D, Liu Y, Hu D, Wei W and Han G: Radiotherapy care during a major outbreak of COVID-19 in Wuhan. Adv Radiat Oncol, 2020. DOI: 10.1016/j.adro.2020.03.004
5 Chinese National Health Commission \& State Administration of Traditional Chinese Medicine. Diagnosis and Treatment Protocol for Novel Coronavirus Pneumonia (In Chinese). Available at: http://www.nhc.gov.cn/yzygj/s7653p/202003/46c9294a7dfe4cef8 0dc7f5912eb1989/files/ce3e6945832a438eaae415350a8ce964.pd $\mathrm{f}$ [Last accessed on May 20th 2020]

Received May 8, 2020

Revised May 20, 2020

Accepted May 21, 2020 